\title{
Modulation of aquaporin gene expression by $n-3$ long-chain PUFA lipid structures in white and brown adipose tissue from hamsters
}

\author{
Paula A. Lopes ${ }^{1 *}$, Rute Martins ${ }^{2,3} \dagger$, Inês Vieira da Silva ${ }^{2,3} \dagger$, Marta S. Madeira ${ }^{1}$, José A. M. Prates ${ }^{1}$ \\ and Graça Soveral ${ }^{2,3 *}$ \\ ${ }^{1}$ CIISA (Centro de Investigação Interdisciplinar em Sanidade Animal), Faculdade de Medicina Veterinária, Universidade de \\ Lisboa, Avenida da Universidade Técnica, Pólo Universitário do Alto da Ajuda, 1300-477 Lisboa, Portugal \\ ${ }^{2}$ Research Institute for Medicines (iMed.ULisboa), Faculdade de Farmácia, Universidade de Lisboa, Avenida Professor Gama \\ Pinto, 1649-003 Lisboa, Portugal \\ ${ }^{3}$ Departamento de Bioquímica e Biologia Humana, Faculdade de Farmácia, Universidade de Lisboa, Avenida Professor \\ Gama Pinto, 1649-003 Lisboa, Portugal
}

(Submitted 20 April 2018 - Final revision received 31 July 2018 - Accepted 6 August 2018)

\section{Abstract}

EPA (20:5n-3) and DHA (22:6n-3) fatty acids have weight-reducing properties with physiological activity depending on their molecular structure - that is, as TAG or ethyl esters (EE). Aquaporins (AQP) are membrane protein channels recognised as important players in fat metabolism, but their differential expression in white adipose tissue (WAT) and brown adipose tissue (BAT), as well as their modulation by dietary $n$-3 long-chain PUFA (LCPUFA) such as EPA and DHA, has never been investigated. In this study, the transcriptional profiles of AQP3, AQP5, AQP7 and selected lipid markers of WAT (subcutaneous and visceral) and BAT (interscapular) from hamsters fed diets containing $n-3$ LCPUFA in different lipid structures such as fish oil (FO, rich in EPA and DHA in the TAG form) and FO-EE (rich in EPA and DHA in the EE form) were used and compared with linseed oil (LSO) as the reference group. A clear effect of fat depot was observed for $A Q P 3$ and leptin $(L E P)$, with the lowest values of mRNA found in BAT relative to WAT. The opposite occurred for PPAR $\alpha$. AQP7 was affected by diet, with FOfed hamsters having higher mRNA levels compared with LSO-fed hamsters. The relative gene expression of $A Q P 5$, adiponectin ( $A D I P O)$, GLUT4 and $P P A R \gamma$ was influenced by both fat tissue and diet. Taken together, our results revealed a differential expression profile of AQP and some markers of lipid metabolism in both WAT and BAT in response to feeding $n$ - 3 LCPUFA in two different structural formats: TAG $v$. EE.

Key words: Aquaporins: Markers of lipid metabolism: $n$-3 Long-chain PUFA: Brown adipose tissue: White adipose tissue

Excessive intake of a Western-style diet rich in SFA and $n-6$ long-chain PUFA (LCPUFA) and poor in $n-3$ LCPUFA is one of the major factors promoting the onset of obesity ${ }^{(1,2)}$. In contrast, EPA $(20: 5 n-3)$ and DHA $(22: 6 n-3)$ acids were reported, more than just once, as having weight-reducing properties ${ }^{(3-5)}$. The physiological activity of $n$-3 LCPUFA may depend on their specific lipid structure. Although not consensual, the TAG form seems to be more bioavailable and, therefore, better absorbed and assimilated than the ethyl esters (EE) form ${ }^{(6)}$.

Adipose tissue plays a central role in regulating energy balance through its metabolic, cellular and endocrine functions. It has been classified into anabolic white adipose tissue (WAT) and catabolic brown adipose tissue $(\mathrm{BAT})^{(7)}$. However, certain differentiated adipocytes can undergo a process known by 'browning', switching its metabolic phenotype from typical WAT to those resembling BAT and altering between anabolic and catabolic states $^{(7)}$. Subcutaneous WAT and visceral WAT are metabolically distinct. Subcutaneous fat expands predominantly by hyperplasia and appears protective through enhanced adipogenic capacity ${ }^{(8)}$. In opposition, visceral fat expansion occurs mainly by hypertrophy with great infiltration of macrophages ${ }^{(9)}$. Visceral adipose tissue activity is also regulated by a great number of glucocorticoid receptors $\beta$-adrenoceptors and a lower number of insulin receptors ${ }^{(10,11)}$. Hence, visceral fat accumulation is important in clinical terms because it is more closely associated with the metabolic syndrome. Conversely, BAT constitutes a metabolically active tissue responsible for non-shivering thermogenesis and depletion of excess energy content ${ }^{(12)}$.

At birth, human newborns have a considerable amount of BAT in their body (1-5\% total body weight, meaning a mass of

Abbreviations: ADIPO, adiponectin; AQP, aquaporins; BAT, brown adipose tissue; EE, ethyl esters; FO, fish oil; LCPUFA, long-chain PUFA; LEP, leptin; LSO, linseed oil; WAT, white adipose tissue.

$\dagger$ These authors contributed equally to this work.

* Corresponding authors: P. A. Lopes, fax +351 213652829, email ampalopes@fmv.ulisboa.pt; G. Soveral, fax +351 217946470, email gsoveral@ff.ulisboa.pt 
35-200 g), which is implicated in heat generation, whereas skeletal muscle is not able to make any movements to produce heat ${ }^{(13)}$. In puberty, this function declines, and is associated with sex-dependent hormones. Recently, BAT was also found to be functionally and metabolically active in adult humans ${ }^{(14)}$, and it is more developed in people exposed to continuous cold, such as outdoor workers ${ }^{(15)}$. In fact, in adults, BAT can amount to $<100 \mathrm{~g}$ in average, which might seem few, but when activated it can be able to consume $3-4 \mathrm{~kg}$ of WAT in 1 year ${ }^{(14)}$. Consequently, BAT appears to be preventive against metabolic disorders associated with obesity ${ }^{(16)}$. In fact, the differential expression of brown adiposity phenotype may, in part, explain why some individuals are more prone to obesity than others. It actually underlies the new concept of metabolically healthy obesity.

Aquaporins (AQP) are a family of transmembrane protein channels that facilitate the permeation of water and small solutes, such as glycerol, across cell membranes ${ }^{(17,18)}$. According to their primary sequence and permeability characteristics, $A Q P$ are divided into three sub-groups: (i) orthodox or classical AQP (AQP0, AQP1, AQP2, AQP4, AQP5, AQP6 and AQP8), primarily water selective ${ }^{(17,18)}$; (ii) aquaglyceroporins (AQP3, AQP7, AQP9 and AQP10), permeable to small uncharged solutes, such as glycerol and urea in addition to water ${ }^{(17-19)}$; (iii) unorthodox or S-AQP (AQP11 and AQP12), found in intracellular membranes, with lower sequence similarity to the other mammalian AQP and suggested as permeable to water (AQP11 and AQP12) and glycerol (AQP12) ${ }^{(20,21)}$

In the adipose tissue, some AQP isoforms may have a pivotal role in controlling fat accumulation ${ }^{(22,23)}$ and have been implicated in obesity and related metabolic complications ${ }^{(24)}$. Among the various mammalian aquaglyceroporins, AQP7 is the most representative glycerol channel and was the first to be detected in human and mouse adipose tissue ${ }^{(25,26)}$. Impaired glycerol transport through AQP-7 (an aquaglyceroporin) leads to glycerol retention within adipose tissue, ultimately leading to acceleration of TAG synthesis and accumulation, which has been correlated to obesity development ${ }^{(25,27,28)}$. However, the presence of other aquaglyceroporins in addition to AQP7 has been more recently reported $^{(23,29)}$. AQP3 was identified in both subcutaneous and visceral human fat depots but with a stronger expression in the stromal vascular tissue adjacent to adipose ${ }^{(23,30,31)}$. Interestingly, AQP3 was the isoform with higher expression in human visceral adipose tissue followed by AQP9 and AQP7, with AQP3 gene expression being found in both fully mature adipocytes and stroma vascular fraction (SVF) cells. $A Q P 3$ is highly expressed in preadipocytes, macrophages and other cells within the SVF (leucocytes, neutrophils, erythrocytes and fibroblasts). Similar to $A Q P 7, A Q P 3$ is up-regulated by catecholamines, suggesting that, together with other aquaglyceroporins, it may be relevant to regulate paracellular transport of glycerol between the bloodstream and the interstitium of WAT ${ }^{(23,24)}$.

More recently, our group detected the expression of $A Q P 5$ in 3T3-L1 adipocytes and showed that besides a role in water transport AQP5 depletion reduces lipid droplets content and impairs adipocyte differentiation ${ }^{(32)}$. However, AQP5 involvement in the obesity mechanisms and the differential expression of these AQP isoforms in WAT (subcutaneous and visceral) and BAT remains unclear.
In this work, we investigated the variability of AQP ( $A Q P 3$, $A Q P 5, A Q P 7)$ gene expression across subcutaneous WAT, visceral WAT and interscapular BAT, which might be associated with adipose tissue depot's own location and their different metabolic functions. In addition, to further investigate whether the body fat reduction properties of $n-3$ LCPUFA in vivo are correlated with AQP expression levels in the adipose tissue, we compared the AQPs' transcriptional profile of WAT (subcutaneous and visceral) and BAT from hamsters fed diets containing specific $n$-3 LCPUFA molecular structures: fish oil (FO, rich in EPA and DHA in the TAG form) and FO-EE (rich in EPA and DHA in the EE form), $v$. linseed oil (LSO, rich in $\alpha$-linolenic acid (ALA), 18:3n-3) used as control. The expression of some markers of lipid metabolism, namely adiponectin (ADIPO), leptin (LEP), GLUT4, PPAR $\alpha$ and PPAR , was also characterised in the different adipose tissue samples.

\section{Methods}

\section{Ethics statement}

The experimental procedures were reviewed by the Ethics Commission of CIISA/FMV and approved by the Animal Care Committee of the National Veterinary Authority (Direcção Geral de Alimentação e Veterinária, Portugal), following the appropriate European Union guidelines (2010/63/EU Directive).

\section{Animals and diets}

Besides having BAT in considerable amounts owing to hibernation, the lipid metabolism of hamsters is more similar to humans than mice and $\operatorname{rats}^{(33,34)}$, thus making it possible to extrapolate results to humans.

In all, 10-week-old Golden Syrian male hamsters were purchased from Charles River (Charles River Laboratories). Hamsters were housed one per cage under a 14/10-h light/dark cycle schedule, synchronised with natural daylight, with controlled temperature of $20-24^{\circ} \mathrm{C}$ in a certified facility at the Faculty of Veterinary, University of Lisbon. During the first week, all animals were fed a standard diet to minimise stress and stabilise all metabolic conditions. After this period, twenty-four hamsters were assigned to three body-weight-matched groups, with eight animals each: the LSO group, rich in ALA, without EPA or DHA, taken as the control group; the FO group, a commercially available oil rich in TAG with EPA and DHA; and the FO-EE group. The final sum of EPA and DHA was identical across FO and FO-EE dietary treatments ${ }^{(6,35)}$. Diets were manufactured by the Experimental Diets Unit from the University of Almería in Spain. The detailed composition of the experimental diets, in terms of proximate chemical composition and fatty acids, has been published elsewhere $^{(6)}$. Briefly, all diets were based on AIN-93M formulation with modified lipid fractions. Each diet contained the following (g/100 g feed): casein (14.0), maize starch (46.6), maltodextrin (15.5), sucrose (10.0), cellulose (5.0), soyabean oil (4.0), L-cystine (0.18), AIN-93 mineral mix (3.5), AIN-93M vitamin mix (1.0), choline bitartrate $(0 \cdot 25)$ and tert-butylhydroquinone (0.0008).

Throughout the trial, hamsters had free access to water and food. Body weight and feed intake were recorded twice a 
week. After 12 weeks of feeding trial, hamsters were fasted for $12 \mathrm{~h}$, weighed before and after the fasting period and euthanised by a mechanical-physical method. Hamsters were placed in a chamber and anaesthetised using a mixture of $20 \%$ of isofluorane in propylene glycol $(\mathrm{v} / \mathrm{v})$ for $30 \mathrm{~s}^{(36)}$, followed by decapitation with a small animal guillotine, in certified ethical conditions to minimise animal suffering. Subcutaneous and visceral WAT from hamsters' pelvic and retroperitoneal anatomical regions, respectively, as well as BAT from hamsters' interscapular region were excised, weighed and stored at $-80^{\circ} \mathrm{C}$ for subsequent gene expression determination.

\section{Adipocyte RNA extraction and complementary DNA synthesis}

Total RNA was isolated and purified from subcutaneous WAT, visceral WAT and BAT using a Qiagen RNeasy lipid tissue mini kit (Qiagen) and stored at $-80^{\circ} \mathrm{C}$. To exclude possible DNA contamination, on-column DNA digestion with the RNase-free DNase set (Qiagen) was performed. All procedures were based on the manufacturer's protocol. The RNA concentration was determined spectrophotometrically at $260 \mathrm{~nm}$ using the NanoDrop1 ND-2000c (ThermoFisher Scientific). The ratios 260/280 and $260 / 230 \mathrm{~nm}$ were also determined to assess the purity of RNA samples and the presence of contaminants. Only samples that fulfill the established quality parameters (260/280 and 260/ $230 \mathrm{~nm}$ ratios were used as purity measurements for protein and solvent presence, respectively, considering ratios between 1.8 and 2.2 ) proceeded to complementary DNA (cDNA) synthesis step. To generate cDNA for quantitative PCR, $750 \mathrm{ng}$ of total RNA was reverse-transcribed for $2 \mathrm{~h}$ at $37^{\circ} \mathrm{C}$ using the firststrand cDNA synthesis kit (NZYtech). Specific primers for realtime quantitative PCR were designed for seven genes ( $A Q P 3$, AQP5, AQP7, ADIPO, GLUT4, PPAR $\alpha$ and PPAR $)$. The DNA sequences of the golden hamster (Mesocricetus auratus) genes were obtained from GenBank and then submitted to the Primer3 software (http://primer3.ut.ee/) to generate primers. Primer3 parameters were set in order to obtain the best pair of primers in size $(20-27 \mathrm{bp})$, melting temperature $\left(50-65^{\circ} \mathrm{C}\right)$, $\%$ GC (50-60\%) and product size range (75-200 bp). The characterisation of the selected genes used in real-time quantitative PCR is described in Table 1.

\section{Real-time quantitative PCR}

Real-time quantitative PCR was performed using PowerUp ${ }^{\mathrm{TM}}$ SYBR $^{\circledR}$ Green Master Mix (Life Technologies) and Xpert Fast SYBR (GRiSP) to amplify AQP ( $A Q P 3,5$ and 7) and markers of lipid metabolism (ADIPO, LEP, GLUT4, PPAR $\alpha$ and PPAR $)$, respectively. The final reaction volume of $20 \mu \mathrm{l}$ was prepared using $10 \mu \mathrm{l}$ of SYBR master mix, $3 \mu \mathrm{l}$ of template cDNA, $2 \mu \mathrm{l}$ of forward and reverse primers and $3 \mu \mathrm{l}$ of molecular-biologygrade water. The reaction was performed on a $\mathrm{CFX} 96^{\mathrm{TM}}$ Real-Time PCR Detection System C1000 (BioRad) consisting of an initial denaturation step at $95^{\circ} \mathrm{C}$ for $3 \mathrm{~min}$, forty-five cycles of denaturation at $95^{\circ} \mathrm{C}$ for $10 \mathrm{~s}$ and annealing/extension at 59 or $62^{\circ} \mathrm{C}$ (for AQP and markers of lipid metabolism, respectively) for $30 \mathrm{~s}$. The relative expression levels were normalised to reference genes (glyceraldehyde-3-phosphate dehydrogenase $(G A P D H)$ and $\beta$-actin $(A C T B)$ for $A Q P 3, A Q P 5$ and $A Q P 7$; ribosomal protein L27 (RPL27) for ADIPO, LEP, GLUT4, PPAR and $P P A R \gamma$ ) and calculated using a variation of the Livak $\operatorname{method}^{(37)}$, corrected for variation in amplification efficiency, as described by Fleige \& Pfaffl $^{(38)}$.

\section{Statistical analysis}

Statistical analyses were carried out with the Statistical Analysis System software, version 9.4 (SAS Institute). All data were

Table 1. Gene-specific primer sequences used for real-time-quantitative PCR

\begin{tabular}{|c|c|c|c|c|}
\hline Gene symbol & Full gene name & GenBank accession no. & Forward/reverse primers & Product size (bp) \\
\hline$A Q P 3$ & Aquaporin-3 & XM_005078855.2 & $\begin{array}{l}\text { F: 5'-CCAACAATGAGCTTATCGTCTCCG-3' } \\
\text { R: 5'-CAGAACACACACGATGAGGGAG-3' }\end{array}$ & 137 \\
\hline$A Q P 5$ & Aquaporin-5 & XM_005067227.2 & $\begin{array}{l}\text { F: 5'-GGTGGTCATGGATCGGTTCAG-3' } \\
\text { R: 5'-GAAGAGCAGGTAGAAGTAGAGCAG-3' }\end{array}$ & 100 \\
\hline$A Q P 7$ & Aquaporin-7 & XM_013119574.1 & $\begin{array}{l}\text { F: 5'-GCAGAGGGAGATGGTACGAGAG-3' } \\
\text { R: 5'-GTCTCCTAGAACCATATGAGCCAC-3' }\end{array}$ & 100 \\
\hline$A D I P O$ & Adiponectin & GQ355976.1 & $\begin{array}{l}\text { F: 5'-CTCTTCACCTTCGACCAGTATCAG-3' } \\
\text { R: 5'-CTGCATAGAGTCCACTGTAATCCC-3' }\end{array}$ & 139 \\
\hline LEP & Leptin & XM_005078071.2 & $\begin{array}{l}\text { F: 5'-CCAAAACCCTCATCAAGACCA-3' } \\
\text { R: 5'-AGCCCAGGAATGAAGTCCAA-3' }\end{array}$ & 106 \\
\hline GLUT4 & $\begin{array}{l}\text { Solute carrier family } 2 \\
\text { (facilitated GLUT), member } 4\end{array}$ & XM_005067520.2 & $\begin{array}{l}\text { F: 5'-ATGGCTGTCGCTGGTTTCTC-3' } \\
\text { R: 5'-AAGCAGGAGGACGGCAAATA-3' }\end{array}$ & 117 \\
\hline PPARa & PPAR $a$ & NM_001281543.1 & $\begin{array}{l}\text { F: 5'-TGAGGAAGCCGTTCTGTGAC-3' } \\
\text { R: 5'-GGTGTCATCTGGATGGTTGC-3' }\end{array}$ & 221 \\
\hline PPARY & PPAR $y$ & XM_013110341.1 & $\begin{array}{l}\text { F: 5'-GAGGGCGATCTTGACAGGAA-3' } \\
\text { R: 5'-GATGGCCACCTCTTTGCTCT-3' }\end{array}$ & 139 \\
\hline \multicolumn{5}{|c|}{ Housekeeping genes } \\
\hline$A C T B$ & $\beta$-Actin & NM_001281595.1 & $\begin{array}{l}\text { F: 5'-GCCAACCGTGAAAAGATGACC-3' } \\
\text { R: 5'-GTACGACCAGAGGCATACAGG-3' }\end{array}$ & 104 \\
\hline$G A P D H$ & $\begin{array}{l}\text { Glyceraldehyde-3-phosphate } \\
\text { dehydrogenase }\end{array}$ & DQ403055.1 & $\begin{array}{l}\text { F: 5'-CAGTATGACTCTACCCATGGCAAG-3' } \\
\text { R: 5'-CAGTAGACTCCACAACATACTCGG-3' }\end{array}$ & 157 \\
\hline$R P L 27$ & Ribosomal protein L27 & XM_005070132.2 & $\begin{array}{l}\text { F: 5'-CATGGGCAAGAAGAAAATCGCC-3' } \\
\text { R: 5'-GTTTCAGGGCTGGGTCTCTAAAG-3' }\end{array}$ & 155 \\
\hline
\end{tabular}


Effect of $n$-3 long-chain PUFA structures on aquaporins
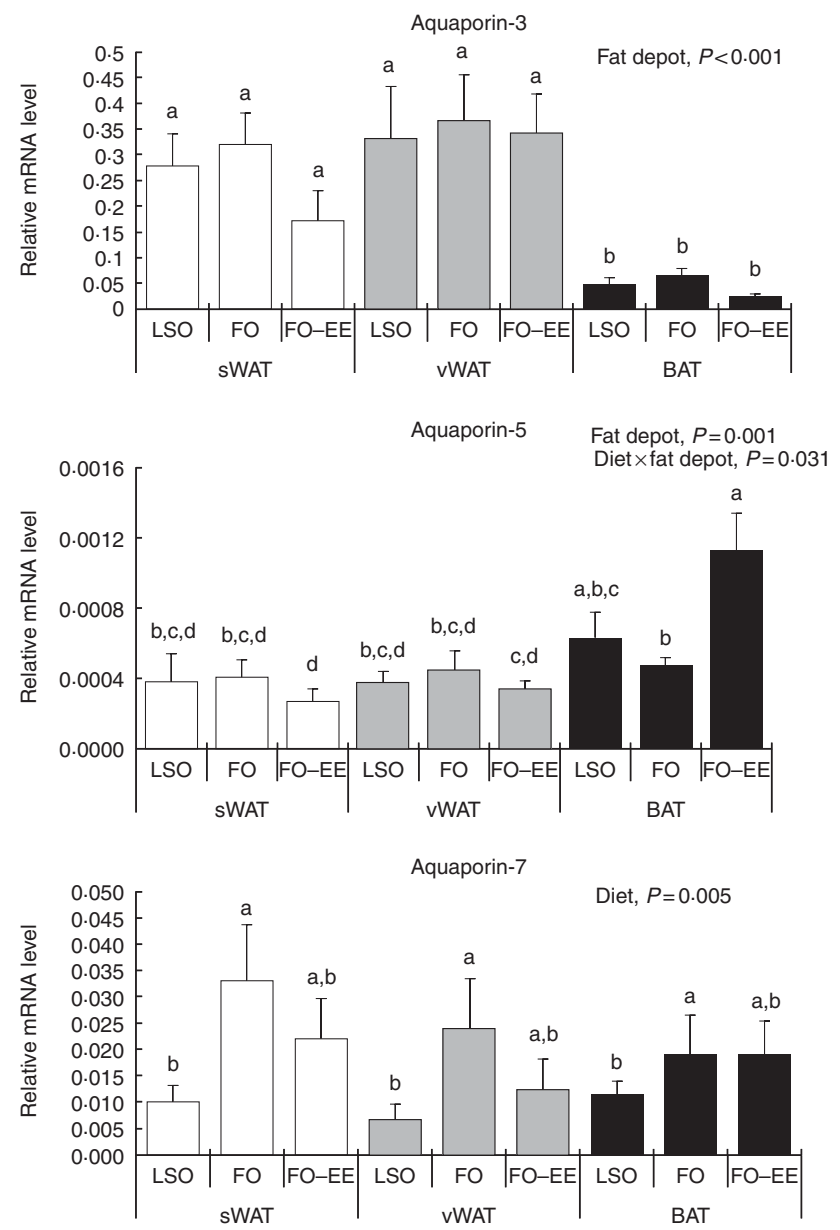

Fig. 1. Effect of fat depot, diet and diet $\times$ fat depot interaction on the relative expression levels of aquaporin-3, aquaporin-5 and aquaporin-7 in the subcutaneous white adipose tissue (sWAT, white bars), visceral white adipose tissue (vWAT, gray bars) and brown adipose tissue (BAT, black bars) from hamsters fed linseed oil (LSO), fish oil (FO) and FO-ethyl esters (EE). Values are means, with their standard errors represented by vertical bars. $\mathrm{a}, \mathrm{b}, \mathrm{c}, \mathrm{d}$ Mean values with unlike letters were significantly different (Tukey's post hoc, $P<0.05)$.

presented as means with their standard errors. Data were checked for normal distribution by Kolmogorov-Smirnov test and variance homogeneity using Proc MIXED with a model including the fat depot and diet as fixed effects and the repeated statement considering the group option to accommodate the variance heterogeneity. This analysis was followed by Tukey's multiple comparisons test. Pearson's correlation coefficients were calculated with the Proc CORR procedure to establish linear relationships among gene expression. $P<0.05$ was considered to be statistically significant.

\section{Results}

Effect of fat depot on gene expression levels of aquaporins and markers of lipid metabolism

In quantitative terms and comparing the transcriptional profile of AQP3, AQP5 and AQP7 in hamster's fat tissues, the levels of AQP3 mRNA were predominant over AQP7 and AQP5 $(A Q P 3>A Q P 7>A Q P 5)$ in all fat depots. A clear effect of fat
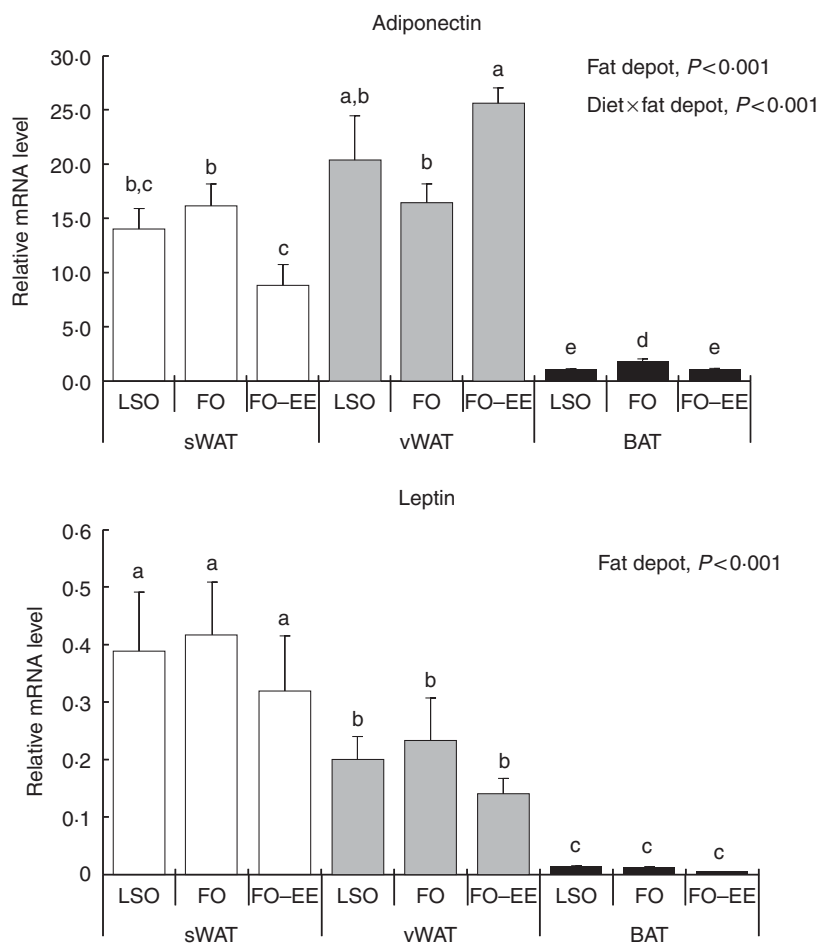

Fig. 2. Effect of fat depot, diet and diet $x$ fat depot interaction on the relative expression levels of adiponectin and leptin in the subcutaneous white adipose tissue (sWAT, white bars), visceral white adipose tissue (vWAT, gray bars) and brown adipose tissue (BAT, black bars) from hamsters fed linseed oil (LSO), fish oil (FO) and FO-ethyl esters (EE). Values are means, with their standard errors represented by vertical bars. ${ }^{a, b, c, d, e}$ Mean values with unlike letters were significantly different (Tukey's post hoc, $P<0.05$ ).

depot was observed for $A Q P 3$ and some markers of lipid metabolism, such as $L E P$ and $P P A R \alpha$, meaning that, regardless of the dietary treatment, the transcriptional profile of this gene was divergent among adipose tissues. This effect of fat depot is most certainly associated with the lower transcriptional profile of $A Q P 3$ in BAT relative to subcutaneous $(P<0 \cdot 001)$ and visceral WAT $(P<0 \cdot 001)$ (Fig. 1). Identical variations were found for LEP in BAT relative to subcutaneous $(P<0.001)$ and visceral $(P<0.001)$ WAT, and also between WAT $(P=0 \cdot 007)$ (Fig. 2$)$. Conversely, PPAR $\alpha$ is highly expressed in BAT in comparison with residual levels of mRNA expression found in both subcutaneous $(P<0.001)$ and visceral $(P<0.001)$ WAT (Fig. 3). The variation pattern between subcutaneous and visceral WAT is similar for some genes, including $A Q P 3, A Q P 5, A Q P 7$ and $L E P$ (Figs 1 and 2, $P>0.05$ ).

\section{Effect of diet on gene expression levels of aquaporins and markers of lipid metabolism}

The only gene affected solely by diet was $A Q P 7$. In fact, increased mRNA levels of this AQP were found in hamsters fed FO relative to LSO $(P=0.008)$ but not in hamsters fed FO-EE relative to LSO $(P=0.050)$, suggesting a positive effect of EPA and DHA when combined as TAG in $A Q P 7$ gene expression (Fig. 1). No changes were detected for $A Q P 7$ gene expression between FO and FO-EE-fed hamsters (Fig. 1, $P=0 \cdot 249$ ). 

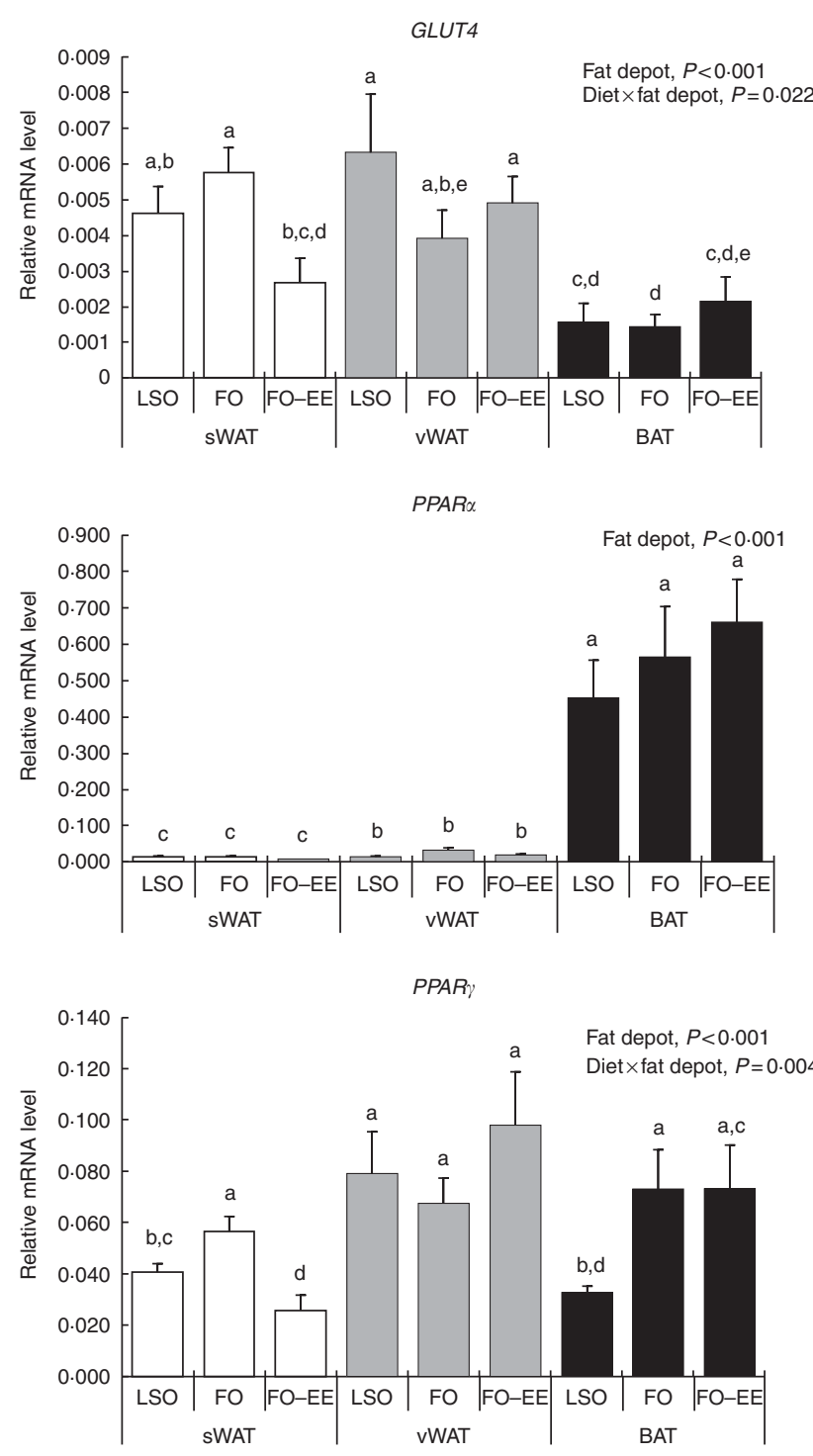

Fig. 3. Effect of fat depot, diet and diet $\times$ fat depot interaction on the relative expression levels of GLUT4, PPARa and PPARY in the subcutaneous white adipose tissue (sWAT, white bars), visceral white adipose tissue (vWAT, gray bars) and brown adipose tissue (BAT, black bars) from hamsters fed linseed oil (LSO), fish oil (FO) and FO-ethyl esters (EE). Values are means, with their standard errors represented by vertical bars. ${ }^{a, b, c, d, e}$ Mean values with unlike letters were significantly different (Tukey's post hoc, $P<0.05$ ).

\section{Interaction effect of fat depot $\times$ diet on gene expression levels of aquaporins and markers of lipid metabolim}

The genes for AQP5, ADIPO, GLUT4 and PPAR $\gamma$ were affected by the interaction between diet and fat, as illustrated in Figs 1-3. An interaction effect between diet and fat was found for the expression level of $A Q P 5$ (Fig. 1, $P=0.031$ ). The reason for this is that BAT presented increased mRNA levels of AQP5 than subcutaneous and visceral WAT when hamsters were fed an FO-EE diet. For ADIPO, higher mRNA levels were observed in the FO diet relative to FO-EE in subcutaneous WAT and BAT, whereas the inverse was found for visceral WAT (Fig. 2, $P<0 \cdot 001)$. GLUT4 was also affected by an interaction between diet and fat (Fig. 3, $P=0.022$ ), which is explained by decreased
mRNA levels in the FO-EE diet in subcutaneous WAT, not verified in BAT and visceral WAT. For PPAR , hamsters fed FO-EE had lower mRNA levels than FO in subcutaneous WAT but identical in visceral WAT and BAT, justifying the interaction detected between diet and fat (Fig. 3, $P=0 \cdot 004$ ).

\section{Pearson's correlation coefficients among genes per fat depot}

Table 2 presents the Pearson's correlation coefficients $(r)$ for gene expression levels in subcutaneous WAT, visceral WAT and BAT. It is noteworthy that that all positive correlations found were moderate $(0 \cdot 7 \geq r \geq 0.3)$. In subcutaneous WAT, $A Q P 3$ was positively correlated with $A D I P O \quad(P<0 \cdot 01)$ and $A Q P 7$ $(P<0 \cdot 01)$. ADIPO correlated positively with $P P A R \gamma(P<0 \cdot 01)$, PPAR $\alpha(P<0 \cdot 01)$ and $L E P(P<0 \cdot 01)$. A positive correlation was observed between GLUT4 and PPAR $(P<0 \cdot 001)$, GLUT4 and PPAR $\alpha(P<0.05)$ and between PPAR $\alpha$ and PPAR $(P<0.01)$ (Table 2). In visceral WAT, fewer correlations were found: $A Q P 3$ was positively correlated with $A Q P 7(P<0.05)$ and also with $A Q P 5 \quad(P<0.05)$ (Table 2). In BAT, $A Q P 7$ correlated positively with PPAR $(P<0.05)$ and LEP $(P<0.05)$. ADIPO was correlated with both PPAR $(P<0.01)$ and LEP $(P<0.05)$. $P P A R \alpha$ correlated with both GLUT4 $(P<0.05)$ and $P P A R \gamma$ $(P<0 \cdot 01)$ (Table 2).

\section{Discussion}

AQP are membrane protein channels recognised as important players in controlling fluid and fat homoeostasis. Although AQP7 has been reported to be involved in obesity, the role of other AQP in fat-related metabolic disorders is still unclear. Moreover, their differential expression in WAT and BAT, as well as their modulation by dietary $n$-3 LCPUFA, such as EPA and DHA in distinct molecular structures, has never been investigated.

In this experimental trial, hamsters' final body weight and weight gain did not vary among dietary groups ${ }^{(6)}$, as well as adipose tissue weight from subcutaneous WAT, visceral WAT and interscapular $\mathrm{BAT}^{(35)}$, as all diets contained the same amount of fat. These data agree with similar values of hamsters' daily feed intake. The abundance of $n-3$ LCPUFA in human fat does not increase notably even after supplementation $^{(39)}$. EPA and DHA are not particularly stored long-term in TAG; rather, they may be partitioned to oxidation pathways or storage in other lipid fractions, such as plasma phospholipids, cell phospholipids and erythrocytes, all of them having a notably higher abundance of both EPA and DHA than adipose tissue ${ }^{(5,6,35)}$.

AQP5 is a classical water channel, whereas AQP3 is an aquaglyceroporin that facilitates permeation of glycerol in addition to water ${ }^{(40)}$. As expected, we found higher levels of $A Q P 3$ expression in WAT in relation to BAT, possibly owing to the main role of WAT in energy storage in the form of TAG. In BAT, AQP3 expression was slightly decreased, although not reaching statistical significance, when hamsters were fed the FO-EE diet, which probably means that the EE molecular form promotes less glycerol efflux in brown adipocytes in 
Table 2. Pearson's correlations coefficients among gene expression levels in subcutaneous white adipose tissue, visceral white adipose tissue and brown adipose tissue

\begin{tabular}{|c|c|c|c|c|c|c|c|}
\hline & $P P A R \gamma$ & PPARa & GLUT4 & LEP & $A D I P O$ & $A Q P 7$ & $A Q P 5$ \\
\hline \multicolumn{8}{|c|}{ Subcutaneous white adipose tissue } \\
\hline$A Q P 3$ & 0.329 & 0.053 & -0.108 & 0.087 & $0.570^{\star \star}$ & $0.660^{\star *}$ & \multirow[t]{8}{*}{0.310} \\
\hline$A Q P 5$ & 0.036 & -0.182 & -0.173 & -0.018 & 0.045 & 0.122 & \\
\hline$A Q P 7$ & 0.335 & 0.059 & 0.084 & -0.048 & 0.340 & & \\
\hline$A D I P O$ & $0.630^{\star *}$ & $0.573^{\star \star}$ & 0.311 & $0.562^{\star \star}$ & & & \\
\hline LEP & 0.269 & 0.036 & 0.035 & & & & \\
\hline GLUT4 & $0.672^{\star \star \star}$ & $0.489^{*}$ & & & & & \\
\hline PPARa & $0.574^{\star *}$ & & & & & & \\
\hline \multirow{2}{*}{\multicolumn{7}{|c|}{ PPARY }} & \\
\hline \multicolumn{7}{|c|}{ Visceral white adipose tissue } & \\
\hline$A Q P 3$ & 0.072 & -0.028 & -0.198 & -0.166 & 0.018 & $0.500^{*}$ & \multirow[t]{8}{*}{0.438} \\
\hline$A Q P 5$ & 0.097 & 0.351 & -0.014 & 0.149 & 0.199 & 0.077 & \\
\hline$A Q P 7$ & 0.361 & -0.044 & -0.089 & 0.380 & -0.024 & & \\
\hline$A D I P O$ & 0.240 & -0.218 & -0.197 & 0.223 & & & \\
\hline LEP & 0.119 & -0.078 & 0.015 & & & & \\
\hline GLUT4 & -0.063 & -0.053 & & & & & \\
\hline PPARa & -0.049 & & & & & & \\
\hline PPARY & & & & & & & \\
\hline \multicolumn{8}{|c|}{ Brown adipose tissue } \\
\hline$A Q P 3$ & 0.012 & -0.182 & -0.420 & -0.194 & 0.192 & 0.332 & \multirow[t]{8}{*}{-0.133} \\
\hline AQP5 & -0.151 & 0.057 & 0.095 & -0.327 & -0.386 & -0.048 & \\
\hline$A Q P 7$ & $0.503^{\star}$ & 0.796 & 0.667 & $0.137^{*}$ & 0.069 & & \\
\hline$A D I P O$ & $0.574^{\star *}$ & 0.302 & 0.015 & $0.530^{\star}$ & & & \\
\hline LEP & 0.129 & 0.244 & 0.280 & & & & \\
\hline GLUT4 & -0.069 & $0.500^{*}$ & & & & & \\
\hline PPARa & $0.594^{* *}$ & & & & & & \\
\hline PPARY & & & & & & & \\
\hline
\end{tabular}

$L E P$, leptin; $A D I P O$, adiponectin; $A Q P$, aquaporins.

${ }^{\star} P<0.05,{ }^{* \star} P<0.01,{ }^{\star \star \star} P<0.001$.

comparison with TAG. Interestingly, when comparing the transcriptional profile of both $\mathrm{AQP}$, an inverse pattern of variation was found for $A Q P 3$ and $A Q P 5$ between $\mathrm{FO}$ and $\mathrm{FO}-\mathrm{EE}$ diets in BAT. Although AQP5 does not contribute to glycerol fluxes, it was shown to be important for adipocyte differentiation $^{(32)}$, and thus a complementary role of these two isoforms for intracellular TAG accumulation cannot be ruled out.

Among the various mammalian aquaglyceroporins, AQP7 is the most representative glycerol channel and was the first to be detected in the adipose tissue from human and rodents ${ }^{(41,42)}$, although predominance of AQP3 in human visceral adipose tissue has been reported ${ }^{(30)}$. In the adipose tissue from hamsters the higher expression was found for $A Q P 3$, rather than for $A Q P 7$. In the overall picture, $A Q P 7$ expression was only affected by diet, with higher levels of mRNA found in the FO dietary treatment in comparison with LSO and FO-EE, suggesting that EPA and DHA when combined as TAG activate the efflux of glycerol from the adipocytes via AQP7, possibly leading to a positive decrease in lipid droplets size. Curiously, the expression of $A Q P 7$ was kept unchanged in all three adipose tissues (there was no fat depot effect), confirming its conservative role in glycerol release from adipocytes that ensure lipid homoeostasis ${ }^{(41)}$.

ADIPO has emerged as the most abundant adipocyte product, thereby redefining adipose tissue as a key component of the endocrine system ${ }^{(43)}$. In obesity, the circulating levels of ADIPO fall while LEP levels rise, suggesting that the regulation of these two adipocyte-derived hormones may be simultaneously influenced by common obesity-related factors ${ }^{(44)}$.
In fact, ADIPO and LEP are adipokines responsible for normal adipocyte phenotype and function ${ }^{(45)}$. The regulation pattern of gene expression of $A D I P O$ in subcutaneous and visceral WAT was similar, perhaps subjective to common control of energy balance. The same does not apply for $L E P$. LEP administration has been shown to down-regulate $A Q P 7$ expression in murine subcutaneous adipocytes ${ }^{(46)}$. We found no correlation (positive or negative) between $L E P$ and $A Q P 7$ mRNA levels in subcutaneous WAT, but instead a positive correlation in BAT that deserves further investigation. In BAT, both ADIPO and LEP showed lower expression, indicating that ADIPO and LEP have in WAT their major contributor. In fact, WAT is known to be the most relevant tissue that accounts for endocrine factors, such as ADIPO and LEP in the bloodstream ${ }^{(47)}$. However, ADIPO production by BAT, although in lower amounts, is well documented $^{(48,49)}$. It is now evident that the LEP synthesis also takes place in BAT, although the physiological role of LEP produced by brown fat is still unclear.

Conversely, PPAR $\alpha$ was highly expressed in BAT, which certainly relates to fatty acid $\beta$-oxidation ${ }^{(50)}$ occurring in brown adipocytes as BAT is primarily a thermogenic organ. In turn, PPAR $\gamma$ stimulates glycerol transport and glycerol phosphorylation $^{(51,52)}$. Moreover, $A Q P 7$ is a direct PPAR $\gamma$ target gene in adipocytes $^{(51,53)}$. This evidence is not supported by the moderate positive correlation found between $A Q P 7$ and $P P A R \gamma$ mRNA levels in BAT, rather than what would be expected to be found in WAT. Furthermore, we have demonstrated that, in subcutaneous WAT, PPAR $\alpha$ and PPAR $\gamma$ expression correlated significantly with the expression of GLUT4, which is an essential 
gene in the lipogenesis pathway ${ }^{(45)}$. These findings demonstrate a relationship between PPAR expression and the expression of other genes of lipid metabolism, and support the concept that $P P A R \alpha$ and $P P A R \gamma$ activators may regulate fatty acid metabolism in the adipose tissue.

Variations of response across fat depots (subcutaneous WAT, visceral WAT and BAT) suggest that the features of adipose tissue responsible for adipocyte cells and differentiation may not be homogeneous, both in nature and distribution ${ }^{(54)}$. Visceral fat accumulation, as supported by PPAR up-regulation, which is the key regulator of adipocytes differentiation, is important in clinical terms because it is more closely linked to the metabolic syndrome owing to its inflammatory features. Conversely, brown adipocytes constitute a metabolically active tissue responsible for non-shivering thermogenesis, depletion of excess energy content being less inflammatory than WAT. These features are in line with PPAR,$A D I P O$ and $L E P$ gene variations. BAT is associated with metabolic health owing to its oxidative capacity and appears to be effective in the protection against metabolic disorders associated with obesity and diabetes. In addition, the structure of combined EPA and DHA as EE reduced the gene expression of $A D I P O, G L U T 4$ and PPAR in subcutaneous WAT, as well as $A D I P O$ in BAT. The only exception was $A D I P O$, whose mRNA levels were found to be increased in the FO-EE diet relative to FO in visceral WAT. On the basis of the current research, the physiological activity of $n-3$ LCPUFA and, in particular, the potential effect of a specific fatty acid depends both on its structure and administration form. There seems to be a difference in the apparent bioavailability of $n-3$ LCPUFA, such that the TAG form is more bioavailable than the EE form ${ }^{(55-57)}$. This interpretation is in line with our own findings. TAG define three fatty acid esterified (bonded) to a glycerol backbone and is the natural molecular form that makes up virtually all fats and oils in both animal and plants species, devoid of toxicological effects and more chemically stable than $\mathrm{EE}^{(58,59)}$. The EE form lacks the glycerol backbone needed to reassemble the TAG structure. We speculate that this could be the major obstacle to the efficient absorption of EE form EPA and DHA, impairing in due course the transcriptional profile of these genes.

\section{Conclusions}

In this study, we reported differential $A Q P 3, A Q P 5$ and $A Q P 7$ gene expression across subcutaneous WAT, visceral WAT and interscapular BAT, which may reflect adipose tissue depot's own location and metabolic function. We also found different patterns of AQP and markers of lipid metabolism expression between WAT (subcutaneous and visceral) and BAT when hamsters were fed specific $n$-3 LCPUFA molecular structures: FO (rich in EPA and DHA in the TAG form) and FO-EE (rich in EPA and DHA in the EE form). Among the AQP isoforms, AQP7 stands out as the more promising target for developing new anti-obesity drugs owing to its conservative role across WAT and BAT.

\section{Acknowledgements}

The authors are grateful to Susana V. Martins for technical assistance in primer use and validation.
This study was supported by the Spanish Ministry of Science and Innovation through 'Structured lipids: novel dietary strategies for improving human health' grant (AGL/25807/2011) and by Fundação para a Ciência e a Tecnologia, Portugal, through projects UID/CVT/00276/2013 and 2016 INOV-20 to CIISA, and UID/DTP/04138/2013 to iMed.ULisboa. I. V. d. S. is the recipient of a PhD fellowship (PD/BD/113634/2015) and M. S. M. of a post-doctoral fellowship (SFRH/BPD/97432/2013). P. A. L. is a post-doctoral researcher from Portugal 2020 project (08/SI/ 3399/2015).

P. A. L. and M. S. M. performed the animal experiment and tissue sampling. I. V. d. S. and R. M. performed the laboratory work. M. S. M. was responsible for statistics. P. A. L. and G. S. were responsible for interpretation of the results and preparation of the manuscript. J. A. M. P. was responsible for the study design. All the authors read and approved the findings of the study.

The authors declare that there are no conflicts of interest.

\section{References}

1. Simopoulos AP (2008) The omega-6/omega-3 fatty acid ratio, genetic variation, and cardiovascular disease. Asia Pac J Clin Nutr 17, 131-134.

2. Ravussin E \& Kozak LP (2009) Have we entered the brown adipose tissue renaissance? Obes Rev 10, 265-268.

3. Calder P (2006) n-3 Polyunsaturated fatty acids, inflammation, and inflammatory diseases. Am J Clin Nutr 83, 1505S1519S.

4. Rodrigues PO, Martins SV, Lopes PA, et al. (2014) Influence of feeding graded levels of canned sardines on the inflammatory markers and tissue fatty acid composition of Wistar rats. BrJ Nutr 112, 309-319.

5. Todorčević M \& Hodson L (2016) The effect of marine derived $n-3$ fatty acids on adipose tissue metabolism and function. J Clin Med 5, 3-27.

6. Bandarra NM, Lopes PA, Martins SV, et al. (2016) Docosahexaenoic acid at the $s n-2$ position of structured triacylglycerols improved $n-3$ polyunsaturated fatty acid assimilation in tissues of hamsters. Nutr Res 36, 452-463.

7. Lee YH, Mottillo EP \& Granneman JG (2014) Adipose tissue plasticity from WAT to BAT and in between. Biochim Biophys Acta 1842, 358-369.

8. Park HT, Lee ES, Cheon YP, et al. (2012) The relationship between fat depot-specific preadipocyte differentiation and metabolic syndrome in obese women. Clin Endocrinol $\mathbf{7 6}$, 59-66.

9. Barbatelli G, Murano I, Madsen L, et al. (2010) The emergence of cold-induced brown adipocytes in mouse white fat depots is determined predominantly by white to brown adipocyte transdifferentiation. Am J Physiol Endocrinol Metab 298, E1244-E1253.

10. Capurso C \& Capurso A (2012) From excess adiposity to insulin resistance: the role of free fatty acids. Vascul Pharmacol 57, 91-97.

11. Fruhbeck G, Mendez-Gimenez L, Fernandez-Formoso JA, et al. (2014) Regulation of adipocyte lipolysis. Nutr Res Rev 27, 63-93.

12. Birerdinc A, Jarrar M, Stotish T, et al. (2013) Manipulating molecular switches in brown adipocytes and their precursors: a therapeutic potential. Prog Lipid Res 52, 51-61.

13. Virtanen KA \& Nuutila P (2011) Brown adipose tissue in humans. Curr Opin Lipidol 22, 49-54. 
14. Virtanen KA, Lidell ME, Orava J, et al. (2009) Functional brown adipose tissue in healthy adults. $N$ Engl J Med 360, 1518-1525.

15. van Marken Lichtenbelt WD, Vanhommerig JW, Smulders NM, et al. (2009) Cold-activated brown adipose tissue in healthy men. N Engl J Med 360, 1500-1508.

16. Smorlesi A, Frontini A, Giordano S, et al. (2012) The adipose organ: white-brown adipocyte plasticity and metabolic inflammation. Obes Rev 13, Suppl. 2, 83-96.

17. Agre P (2004) Aquaporin water channels (Nobel Lecture). Angew Chem Int Ed Engl 43, 4278-4290.

18. Verkman AS (2005) More than just water channels: unexpected cellular roles of aquaporins. J Cell Sci 118, 3225-3232.

19. Rojek A, Praetorius J, Frøkiaer J, et al. (2008) A current view of the mammalian aquaglyceroporins. Annu Rev Physiol 70, 301-327.

20. Ishibashi K, Tanaka Y \& Morishita Y (2014) The role of mammalian superaquaporins inside the cell. Biochim Biophys Acta 1840, 1507-1512.

21. Madeira A, Fernández-Veledo S, Camps M, et al. (2014) Human aquaporin-11 is a water and glycerol channel and localizes in the vicinity of lipid droplets in human adipocytes. Obesity (Silver Spring) 22, 2010-2017.

22. Fruhbeck G (2005) Obesity: aquaporin enters the picture. Nature 438, 436-438.

23. Rodríguez A, Catalan V, Gomez-Ambrosi J, et al. (2011) Aquaglyceroporins serve as metabolic gateways in adiposity and insulin resistance control. Cell Cycle 10, 1548-1556.

24. da Silva IV, Rodrigues JS, Rebelo I, et al. (2018) Revisiting the metabolic syndrome: the emerging role of aquaglyceroporins. Cell Mol Life Sci 75, 1973-1988.

25. Kishida K, Kuriyama H, Funahashi T, et al. (2000) Aquaporin adipose, a putative glycerol channel in adipocytes. $J$ Biol Chem 275, 20896-20902.

26. Kondo H, Shimomura I, Kishida K, et al. (2002) Human aquaporin adipose (AQPap) gene. Genomic structure, promoter analysis and functional mutation. Eur J Biochem 269, 1814-1826.

27. Hibuse T, Maeda N, Funahashi T, et al. (2005) Aquaporin 7 deficiency is associated with development of obesity through activation of adipose glycerol kinase. Proc Natl Acad Sci US A 102, 10993-10998.

28. Madeira A, Camps M, Zorzano A, et al. (2013) Biophysical assessment of human aquaporin-7 as a water and glycerol channel in 3T3-L1 adipocytes. PLOS ONE 8, e83442.

29. Laforenza U, Scaffino MF \& Gastaldi G (2013) Aquaporin-10 represents an alternative pathway for glycerol efflux from human adipocytes. PLOS ONE $\mathbf{8}$, e54474.

30. Rodriguez A, Catalan V, Gomez-Ambrosi J, et al. (2011) Insulin- and leptin-mediated control of aquaglyceroporins in human adipocytes and hepatocytes is mediated via the PI3K/ Akt/mTOR signaling cascade. J Clin Endocrinol Metab 96, E586-E597.

31. Miranda M, Escote X, Ceperuelo-Mallafre V, et al. (2010) Paired subcutaneous and visceral adipose tissue aquaporin-7 expression in human obesity and type 2 diabetes: differences and similarities between depots. J Clin Endocrinol Metab 95, 3470-3479.

32. Madeira A, Mósca AF, Moura TF, et al. (2015) Aquaporin-5 is expressed in adipocytes with implications in adipose differentiation. IUBMB Life 67, 54-60.

33. Yin W, Carballo-Jane E, McLaren DG, et al. (2012) Plasma lipid profiling across species for the identification of optimal animal models of human dyslipidemia. J Lipid Res 53, 51-65.
34. Dalboge LS, Pedersen PJ, Hansen G, et al. (2015) A hamster model of diet-induced obesity for preclinical evaluation of anti-obesity, anti-diabetic and lipid modulating agents. PLOS ONE 10, 634-648.

35. Lopes PA, Bandarra NM, Martins SV, et al. (2017) Docosahexaenoic acid (DHA) at the $s n-2$ position of triacylglycerols increases DHA incorporation in brown, but not in white adipose tissue, of hamsters. Int J Food Sci Nutr 69, 458-471.

36. Itah R, Gitelman I \& Davis C (2004) A replacement for methoxyflurane (Metofane) in open-circuit anaesthesia. Lab Anim 38, 280-285.

37. Livak KJ \& Schmittgen TD (2001) Analysis of relative gene expression data using real-time quantitative PCR and the 2(-Delta Delta C(T)) method. Methods 25, 402-408.

38. Fleige S \& Pfaffl MW (2006) RNA integrity and the effect on the real-time qRT-PCR performance. Mol Aspects Med 27, 126-139.

39. Baylin A, Kabagambe EK, Siles X, et al. (2002) Adipose tissue biomarkers of fatty acid intake. Am J Clin Nutr 76, 750-757.

40. Madeira A, Moura TF \& Soveral G (2015) Aquaglyceroporins: implications in adipose biology and obesity. Cell Mol Life Sci 72, 759-771.

41. da Silva IV \& Soveral G (2017) Aquaporins in obesity. Adv Exp Med Biol 969, 227-238.

42. Fruhbeck G, Catalan V, Gomez-Ambrosi J, et al. (2006) Aquaporin-7 and glycerol permeability as novel obesity drugtarget pathways. Trends Pharmacol Sci 27, 345-347.

43. Cao H (2014) Adipocytokines in obesity and metabolic disease. J Endocrinol 220, 47-59.

44. Bastard JP, Maachi M, Lagathu C, et al. (2006) Recent advances in the relationship between obesity, inflammation, and insulin resistance. Eur Cytokine Netw 17, 4-12.

45. Cowherd RM, Lyle RE \& McGehee RE Jr (1999) Molecular regulation of adipocyte differentiation. Semin Cell Dev Biol 10, 3-10.

46. Rodríguez A, Moreno NR, Balaguer I, et al. (2015) Leptin administration restores the altered adipose and hepatic expression of aquaglyceroporins improving the non-alcoholic fatty liver of ob/ob mice. Sci Rep 5, 12067.

47. Wang GX, Zhao XY \& Lin JD (2015) The brown fat secretome: metabolic functions beyond thermogenesis. Trends Endocrinol Metab 26, 231-237.

48. Zhang Y, Matheny M, Zolotukhin S, et al. (2002) Regulation of adiponectin and leptin gene expression in white and brown adipose tissues: influence of beta3-adrenergic agonists, retinoic acid, leptin and fasting. Biochim Biophys Acta 1584, $115-122$.

49. Iacobellis G, Di Gioia C, Petramala L, et al. (2013) Brown fat expresses adiponectin in humans. Int J Endocrinol 2013, 126751.

50 Kersten S, Desvergne B \& Wahli W (2000) Roles of PPARs in health and disease. Nature 405, 421-424.

51. Guan HP, Li Y, Jensen MV, et al. (2002) A futile metabolic cycle activated in adipocytes by antidiabetic agents. Nat Med 8, 1122-1128.

52. Tordjman J, Khazen W, Antoine B, et al. (2003) Regulation of glyceroneogenesis and phosphoenolpyruvate carboxykinase by fatty acids, retinoic acids and thiazolidinediones: potential relevance to type 2 diabetes. Biochimie 85, 1213-1218.

53. Kishida K, Shimomura I \& Nishizawa H (2001) Enhancement of the aquaporin adipose gene expression by a peroxisome proliferator-activated receptor gamma. J Biol Chem 276, 48572-48579.

54. Peirce V, Carobbio S \& Vidal-Puig A (2014) The different shades of fat. Nature 510, 76-83. 
55. Lawson LD \& Hughes BG (1988) Absorption of eicosapentaenoic acid and docosahexaenoic acid from fish oil triacylglycerols or fish oil ethyl esters co-ingested with a high-fat meal. Biochem Biophys Res Comm 156, 960-963.

56. Lawson LD \& Hughes BG (1988) Human absorption of fish oil fatty acids as triacylglycerols, free acids, or ethyl esters. Biochem Biophys Res Comm 152, 328-335.

57. Beckermann B, Beneke M \& Seitz I (1990) Comparative bioavailability of eicosapentaenoic acid and docosahexaenoic acid from triglycerides, free fatty acids and ethyl esters in volunteers. Arzneimittelforschung 40, 700-704.

58. Song JH, Inoue Y \& Miyazawa T (1997) Oxidative stability of docosahexaenoic acid containing oils in the form of phospholipids, triacylglycerols, and ethyl esters. Biosci Biotechnol Biochem 61, 2085-2088.

59. Yoshii H, Furuta T, Siga H, et al. (2002) Autoxidation kinetic analysis of docosahexaenoic acid ethyl ester and docosahexaenoic triglyceride with oxygen sensor. Biosci Biotechnol Biochem 66, 749-753. 